\title{
ON A BOUNDED INCREASING POWER SERIES
}

\author{
P. B. KENNEDY AND P. SZÜSZ
}

Recently [1] H. S. Shapiro has shown that if $k$ is a positive integer, $\beta=1 /(2 k)$ and $\alpha=1-\beta$, and if $a_{0}=0, a_{n}=n^{-\alpha} \cos n^{\beta}$ for $n=1,2,3, \cdots$, then the function

$$
f(x)=\sum_{0}^{\infty} a_{n} x^{n}
$$

has bounded variation on $[0,1)$ but the series

$$
\sum_{0}^{\infty} a_{n}
$$

is divergent. Given any $\epsilon>0$ we may by choosing $k$ large enough ensure that

$$
a_{n}=O\left(n^{-1+c}\right) \text {. }
$$

However the series (2) is certainly Abel summable and so the stronger condition

$$
a_{n}=O\left(n^{-1}\right)
$$

would imply the convergence of (2), by Littlewood's Tauberian theorem. Thus Shapiro's example shows that we cannot weaken (4) to a condition of the form (3) in Littlewood's theorem by making the compensating assumption that $f$ has bounded variation on $[0,1)$.

In this note we prove a stronger negative result than that of Shapiro.

TheORem. Let $\phi(n)$ be positive for all positive integers $n$, and let $\phi(n) \uparrow \infty$ as $n \rightarrow \infty$. Then there is a function $f$ of the form (1) which is increasing and bounded on $[0,1)$ and for which (2) is divergent although

$$
\left|a_{n}\right|<n^{-1} \phi(n) \text { for all } n \geqq 1 \text {. }
$$

Proof. Let $\left\{n_{k}\right\}(k=1,2,3, \cdots)$ be an increasing sequence of integers satisfying

$$
\phi\left(n_{k}\right)>k^{2} \quad(k \geqq 1) .
$$

Put

Received by the editors June 28, 1965. 
(7) $\quad a_{n}=\left\{\begin{array}{rll}k^{2} / n & \text { if } \quad k^{2} n_{k} \leqq n<\left(k^{2}+1\right) n_{k}, & k=1,2,3, \cdots \\ -k^{2} / n & \text { if }\left(k^{2}+1\right) n_{k} \leqq n<\left(k^{2}+2\right) n_{k}, & k=1,2,3, \cdots \\ 0 & \text { for all other values of } n . & \end{array}\right.$

(7) defines $a_{n}$ uniquely for all $n \geqq 0$; and if the summation is over the range $k^{2} n_{k} \leqq n<\left(k^{2}+1\right) n_{k}$, then

$$
\sum a_{n}=k^{2} \sum n^{-1}>k^{2} n_{k} /\left\{\left(k^{2}+1\right) n_{k}\right\} \rightarrow 1,
$$

so that (2) is divergent. Moreover for each value of $n$, either $a_{n}=0$ or else by (7) there is a positive integer $k$ such that

$$
n\left|a_{n}\right|=k^{2}, \quad k^{2} n_{k} \leqq n,
$$

and so by (6) and the fact that $\phi$ is increasing,

$$
n\left|a_{n}\right|<\phi\left(n_{k}\right) \leqq \phi(n),
$$

which proves (5).

It remains to show that $f$, defined by (1), is increasing and bounded on $[0,1)$. In fact, by $(7), f(x)=\sum_{1}^{\infty} f_{k}(x)$, where

$$
f_{k}(x)=k^{2} \sum_{1} x^{n} / n-k^{2} \sum_{2} x^{n} / n,
$$

$\sum_{1}$ being taken over the range $k^{2} n_{k} \leqq n<\left(k^{2}+1\right) n_{k}$, and $\sum_{2}$ over the range $\left(k^{2}+1\right) n_{k} \leqq n<\left(k^{2}+2\right) n_{k}$. It is easy to see that $f_{k}^{\prime}(x) \geqq 0$ for all $x$ on $[0,1)$, and so $f^{\prime}(x) \geqq 0$, whence $f$ is increasing on $[0,1)$. Also, when $x=1, \sum_{1}$ consists of $n_{k}$ terms, each at most as big as $1 /\left(k^{2} n_{k}\right)$, and $\sum_{2}$ consists of $n_{k}$ terms, each at least as big as $1 /\left\{\left(k^{2}+2\right) n_{k}\right\}$, and so

$$
f_{k}(1) \leqq 1-k^{2} /\left(k^{2}+2\right) \sim 2 / k^{2} .
$$

Therefore $f(1-0)=\sum_{1}^{\infty} f_{k}(1)<+\infty$. Thus $f$ is increasing and bounded on $[0,1)$, and this proves the theorem.

\section{REFERENCE}

1. H. S. Shapiro, A remark concerning Littlewood's Tauberian theorem, Proc. Amer. Math. Soc. 16 (1965), 258-259.

UNIVERSITY OF YORK, ENGLAND AND Pennsylvania State University 